\title{
Enhancement of the $Q$ value of a microring resonator by introducing curved photonic crystal waveguides
}

\author{
Jun-ichiro Sugisaka, ${ }^{1,2, *}$ Noritsugu Yamamoto, ${ }^{1}$ Makoto Okano, ${ }^{1}$ Kazuhiro Komori, ${ }^{1}$ and Masahide Itoh ${ }^{2}$ \\ ${ }^{1}$ Photonics Research Institute, National Institute of Advanced Industrial Science and Technology, \\ 1-1-1 Umezono, Tsukuba, Ibaraki, Japan \\ ${ }^{2}$ Applied Physics Laboratory, University of Tsukuba, 1-1-1 Tennoudai, Tsukuba, Ibaraki, Japan \\ *Corresponding author: jun-ichiro.sugisaka@aist.go.jp
}

Received January 11, 2012; revised March 14, 2012; accepted April 17, 2012; posted April 27, 2012 (Doc. ID 161340); published June 12, 2012

\begin{abstract}
The aim of this work is to enhance the $Q$ value of a photonic crystal microring resonator that can be used to buffer optical signals (in a pulse train). A conventional photonic crystal microring resonator having $60^{\circ}$ bends exhibits poor confinement of light due to the radiation losses at the bending points. Hence, we replaced conventional bent waveguides with circularly curved ones. The $Q$ value of the resulting ring resonator is measured, and we demonstrate, as an example, an eightfold enhancement of the $Q$ value at a wavelength of $1340 \mathrm{~nm}$. Finally, we also estimate the $Q$ value of a ring waveguide that excludes the optical coupling between the input/output waveguides, showing that the replacement of a sharply bent waveguide by a curved one increases the $Q$ value by up to 5.2 times at a wavelength of $1340 \mathrm{~nm}$. (c) 2012 Optical Society of America
\end{abstract}

OCIS codes: $\quad 050.5298,210.4680$.

\section{INTRODUCTION}

Optical buffer memories that can store an optical pulse train are required for the development of optical packet switching, and various approaches have been proposed to develop such memories. A ring resonator consisting of an optical fiber loop [1] can store many optical pulses. However, because optical fibers cannot bend sharply and have no slow-light frequency band, they are difficult to miniaturize and integrate. As a solution, a photonic crystal ring resonator has been proposed. A photonic crystal $[2,3]$ is an optical material with a periodic refractive index distribution. Photonic crystal optical devices can be fabricated on semiconductor wafers, and they can be easily integrated with miniature optical circuits.

A ring resonator consists of a ring waveguide and a $2 \times 2$ optical switch, as sketched in Fig. 1(a). The ring waveguide is a closed loop that can be made of a dielectric waveguide [4-7], a two-dimensional photonic crystal [8-10], a combination dielectric waveguide and photonic crystal [11], or a onedimensional photonic crystal [12]. Ring waveguides have been used for laser [8] and wavelength filters $[\underline{4}-\underline{7}, \underline{10}]$. A directional coupler is a pair of parallel waveguides, realizing a $2 \times 2$ optical switch that can change the optical paths between the so-called bar and cross states. In the bar state, the waveguide is optically decoupled, and any input light is directly output again. In contrast, in the cross state, light is coupled into or out of the waveguide. Such a directional coupler can be fabricated from a photonic crystal [13,14], resulting in considerable miniaturization due to its advantage of anomalous dispersion [15].

An input optical pulse train is guided into a ring waveguide by a cross-state directional coupler and stored by switching the directional coupler to the bar state. The ring waveguide, whose cladding consists of a photonic crystal, not only provides strong confinement of light but also produces relatively low group velocities compared with an optical fiber.
A photonic crystal can also provide nearly zero group velocity, thereby achieving a long pulse delay even for short waveguides, resulting in a small-size buffer circuit.

We previously fabricated a hexagonal microring waveguide $[16,17]$. Its $Q$ value was low, due to radiation losses at the sharp bends. A sharply bent waveguide that can confine light using a photonic bandgap has been reported [18]. In triangular-lattice two-dimensional photonic crystal slabs, however, light is radiated and reflected from the sharp bend points [19]. In a similar fashion, our hexagonal ring resonator exhibits bright spots at the six corners, resulting in a low $Q$ value. In addition, the reflections produce Fabry-Perot interference, which distorts the pulse shape. To solve these problems, we have developed circularly curved waveguides in which the radiation and reflection is considerably reduced [20]. The circularly curved waveguide is designed by bending an ordinary W1 straight waveguide along a circular arc. Spectra (i) and (ii) in Fig. 2 are experimentally obtained transmitted-intensity spectra of photonic crystal waveguides having two $60^{\circ}$ sharp bends and circular bends. Since the difference in the waveguide structure is only the shape at the bending part, the difference in the transmitted intensity corresponds to that of the bent waveguides. By replacing the sharply bent waveguides by the circularly bent waveguides, the transmitted intensity at the shorter wavelength side [region (a) in Fig. 2] is seen to markedly increase. For example, the transmitted intensity of the circular waveguide at around $1340 \mathrm{~nm}$ is about twice as high as that for the sharply bent waveguide. At the longer wavelength side [region (b) in Fig. 2], the spectrum of sharply bent waveguides fluctuates with a period of around $2 \mathrm{~nm}$. This fluctuation is caused by the Fabry-Perot resonance between two bending points. In contrast, the fluctuation in the circularly bent waveguide is depressed. This shows that the replacement of the bent waveguide is effective for the reduction of backreflection in the bent waveguide. 


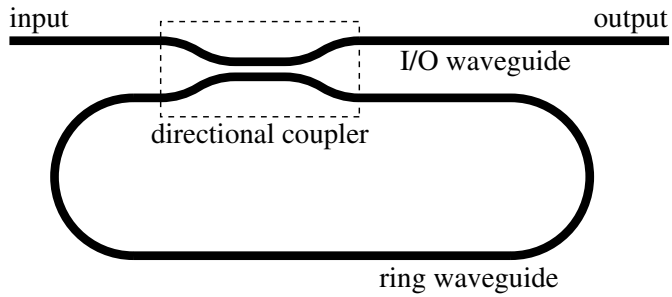

(a)

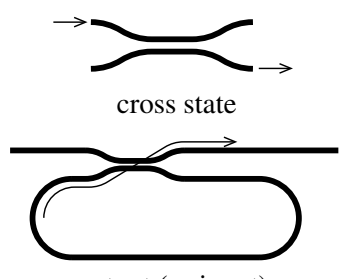

output (or input)

(b)

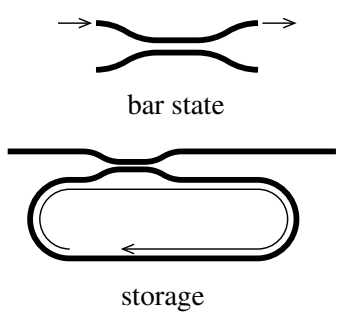

(c)

Fig. 1. (a) Schematic of the ring resonator. It consists of an input/ output (I/O) waveguide and a ring waveguide. These waveguides are optically connected by a directional coupler. (b) The directional coupler in the cross state. Light in the ring waveguide is extracted from or introduced into the ring waveguide. (c) The directional coupler in the bar state. Light in the ring waveguide is confined.

In this study, we design a circular ring resonator in which all sharply bent waveguides are replaced by circularly curved waveguides. We also fabricate ring waveguides having sharp bends (conventional resonators) and circular bends, and we measure the transmitted-intensity spectra to compare the resonance characteristics. In particular, we make comparisons in terms of the $Q$ value, which indicates the strength of light confinement in the ring waveguide, and discuss the effect of the replacement.

\section{DESIGN AND FABRICATION OF THE CIRCULAR RING RESONATOR}

Schematics of hexagonal (conventional) and designed circular ring cavities are shown in Fig. 3. The photonic crystals are fabricated on $190 \mathrm{~nm}$ thick GaAs slabs, and circular air holes are arranged in a triangular lattice with a lattice constant of

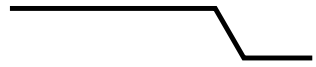

(i) sharp bend

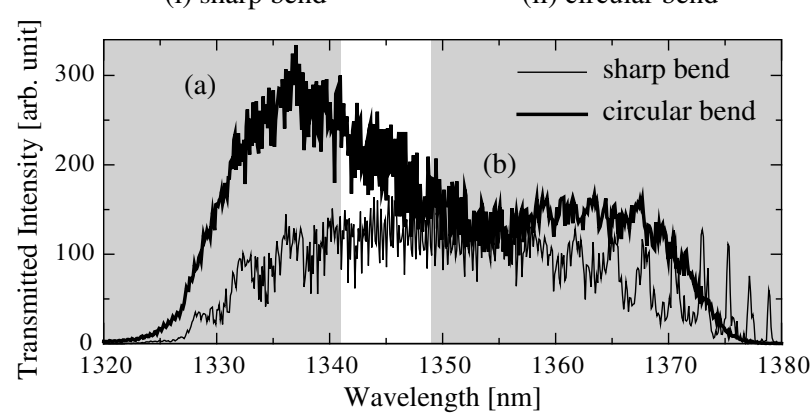

Fig. 2. Experimentally obtained transmitted-intensity spectra of photonic crystal waveguides having (i) sharp bends and (ii) circular bends. Replacing the sharp bends by circular bends increases the transmitted intensity, especially at (a) the shorter wavelength side. (b) At the longer wavelength side, the fluctuation due to the Fabry-Perot resonance has been decreased for circular bends.
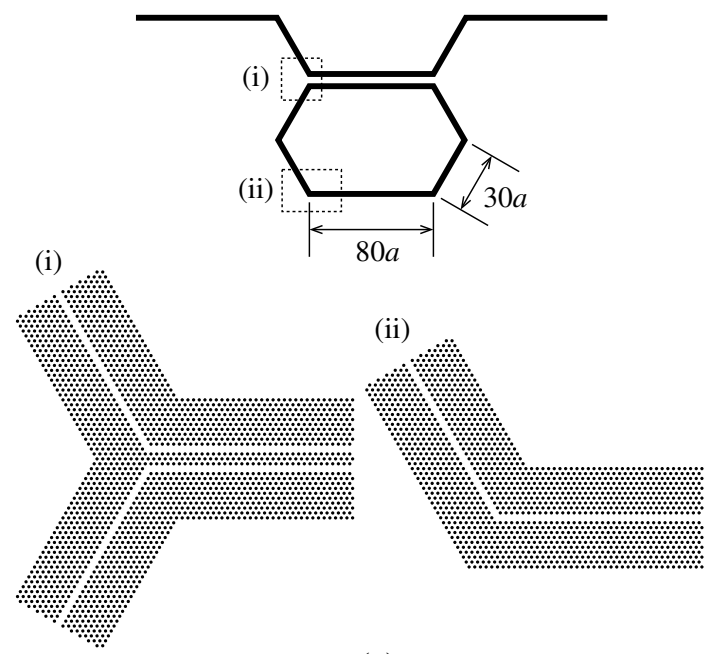

(a)

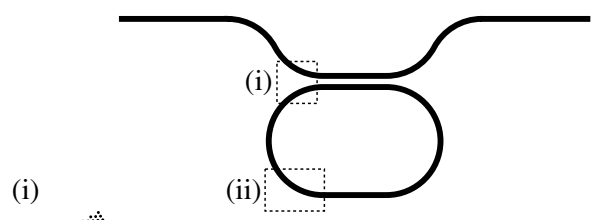

(ii)
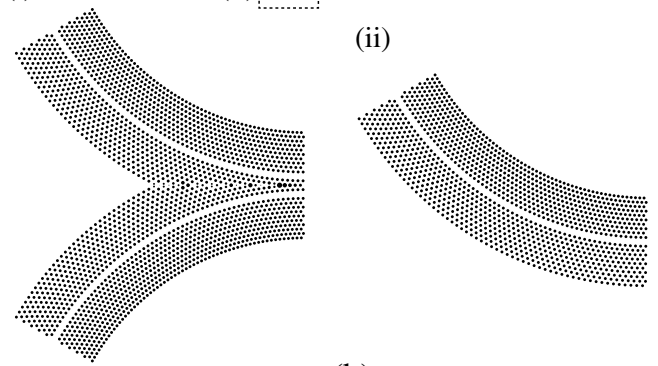

(b)

Fig. 3. Geometry of (a) a conventional hexagonal ring resonator and (b) a circularly curved ring resonator. The patterns of air holes in the dashed boxes are shown in panels (i) and (ii).

$a=390 \mathrm{~nm}$. The radius of the air holes is $0.29 a$. The directional coupler, connecting the input/output (I/O) and ring waveguides, consists of a coupled waveguide separated by three rows of air holes. The length of the directional coupler was set to $80 a$. Both ends of the coupled waveguide are connected to separating waveguides. The separating waveguide for the circular ring resonator consists of a pair of curved waveguides, located at position (i) in Fig. 3(b). The separating waveguide for the hexagonal ring resonator is a Y-shaped separator, sketched in panel (i) of Fig. 3(a). The lengths of the hexagonal and circular waveguides are $280 a$ and $520 a$, respectively. Their curvature, that is, the degree of bend per lattice constant, is 1.0. Both ends of the external waveguides have planar solid immersion lenses [21] to prevent Fabry-Perot interference from reflection at the input and output ports of the device.

The patterns were fabricated lithographically in a resist layer over a GaAs/AlGaAs epitaxial wafer using an electron beam and transferred to the top GaAs layer by dry etching. Then the sacrificial AlGaAs layer under it was removed by wet etching, yielding an air-bridged two-dimensional photonic crystal. To perform measurements on it, light from a tunable laser was input using a collimated lensed fiber to excite the 


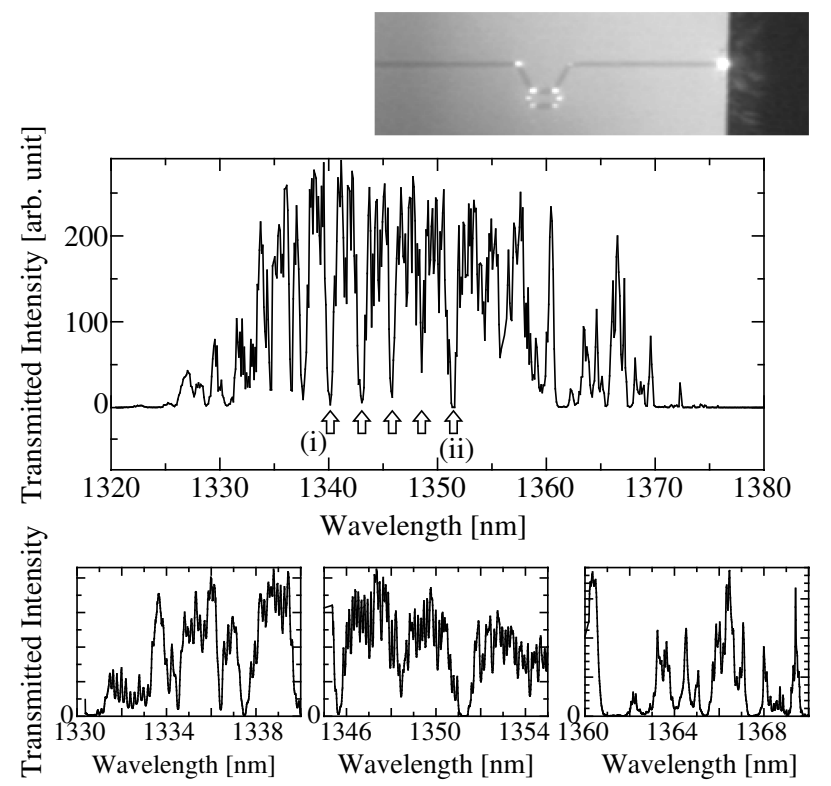

Fig. 4. Transmitted-intensity spectrum for the hexagonal ring resonator. The upper spectrum is scanned over the entire transmission band of the photonic crystal at intervals of $0.1 \mathrm{~nm}$, and arrows indicate resonance peaks. Peaks (i) and (ii) are used to evaluate the $Q$ value. The lower three graphs are scanned in steps of $0.01 \mathrm{~nm}$. The photograph shows the surface of the device when light at the resonant wavelength $(1337.59 \mathrm{~nm})$ is incident.

transverse electric (TE) mode. The output light was picked up by a lensed fiber, and the transmitted intensity was measured using a power meter. The resulting spectra for the hexagonal and circular ring cavities are shown in Figs. 4 and 5, respectively. We observed large peaks in these spectra, which are shown by arrows in the figures.
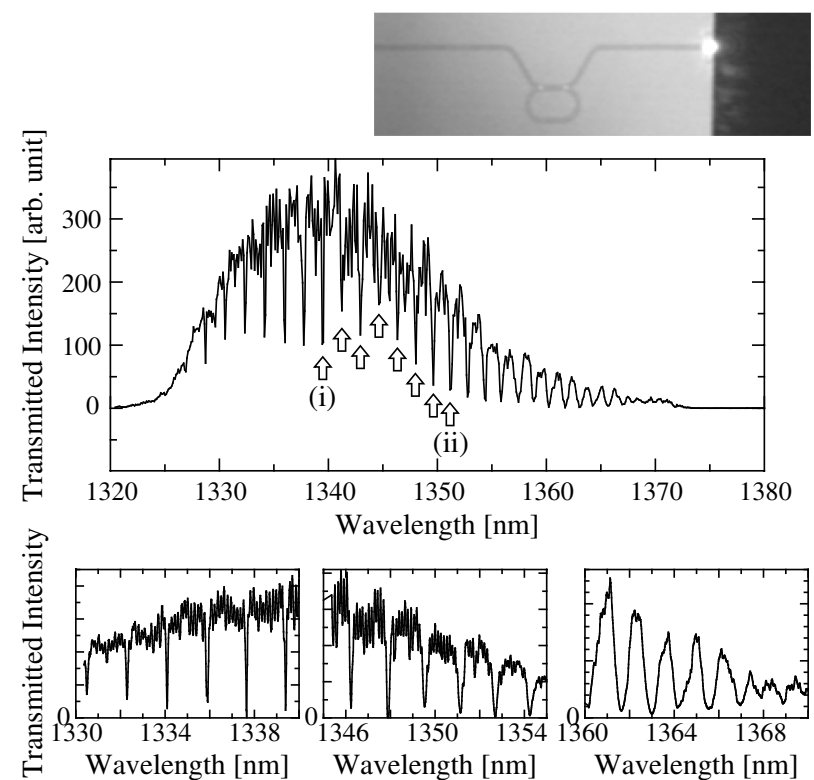

Fig. 5. Transmitted-intensity spectrum for the circular ring resonator. The upper spectrum is scanned over the entire transmission band of the photonic crystal at intervals of $0.1 \mathrm{~nm}$, and arrows indicate resonance peaks. Peaks (i) and (ii) are used to evaluate the $Q$ value. The lower three graphs are scanned in steps of $0.01 \mathrm{~nm}$. The photograph shows the surface of the device when light at the resonant wavelength $(1339.08 \mathrm{~nm})$ is incident.

\section{NUMERICALLY SIMULATED SPECTRA OF RING RESONATORS}

To verify whether the resonant peaks can be attributed to resonance in the ring resonator, we numerically simulated the transmittance spectra of the ring resonators. The transmittance spectrum of a ring resonator, which consists of a directional coupler and an optical fiber loop, has been formulated [22]. The transmittance for a photonic crystal ring resonator is also calculated in the same way:

$I(\omega)=\frac{1}{4}\left|\left(e^{i k_{e} L_{\mathrm{DC}}}+e^{i k_{o} L_{\mathrm{DC}}}\right)+\frac{\left.\left(e^{i k_{e} L_{\mathrm{DC}}}-e^{i k_{o} L_{\mathrm{DC}}}\right)^{2} e^{\left(-\gamma+i k_{\mathrm{wg}} L_{\mathrm{ring}}\right.}\right)}{2-\left(e^{i k_{e} L_{\mathrm{DC}}}+e^{i k_{0} L_{\mathrm{DC}}}\right) e^{\left(-\gamma+i k_{\mathrm{wg}} L_{\text {ring }}\right)}}\right|^{2}$,

where $k_{e}$ and $k_{o}$ are wavenumbers of even and odd modes of the directional coupler and $k_{\mathrm{wg}}$ is a wavenumber for the ring waveguide. $L_{\text {ring }}$ and $L_{\mathrm{DC}}$ are the lengths of the ring waveguide and the directional coupler. $\gamma$ expresses the attenuation in the ring waveguide. The wavenumbers $k_{e}, k_{o}$, and $k_{\mathrm{wg}}$ are obtained from band diagrams given by a three-dimensional finite-difference time-domain method. We assume that $k_{\mathrm{wg}}$ is constant in the ring waveguide and also equal to the wavenumber of the photonic crystal straight waveguide. The band diagrams of the photonic crystal slab waveguide (straight), for even and odd modes of the directional coupler, are shown in Figs. 6(a) and $6(\mathrm{~b})$. Considering the fabrication error of the photonic crystal, slab thickness, and the error in refractive index, we shifted the band diagram in Figs. 6(a) and 6(b) so that the cutoff wavelength at the longer wavelength side (near $1370 \mathrm{~nm}$ ) matches the experimentally measured spectra shown in Fig. 2. The calculated spectra of ring resonators with $L_{\text {ring }}=280 a$ and $L_{\text {ring }}=520 a$ are shown in Figs. $7(\mathrm{a})$ and $\underline{7(\mathrm{~b})}$. The attenuation parameter $\gamma$ is set to 0.1 or 0.5 , which corresponds to small and

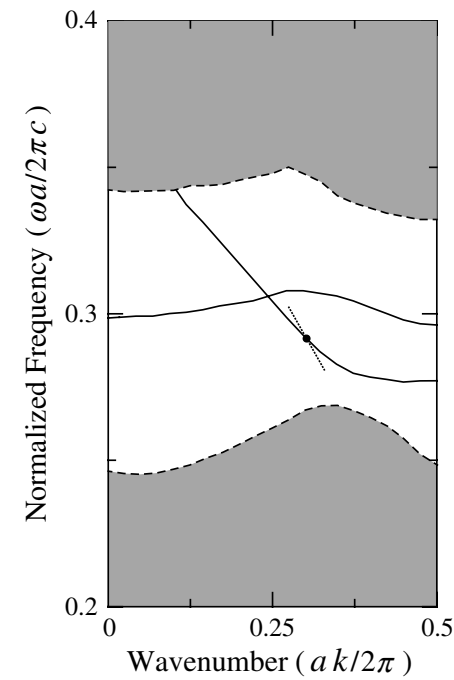

(a)

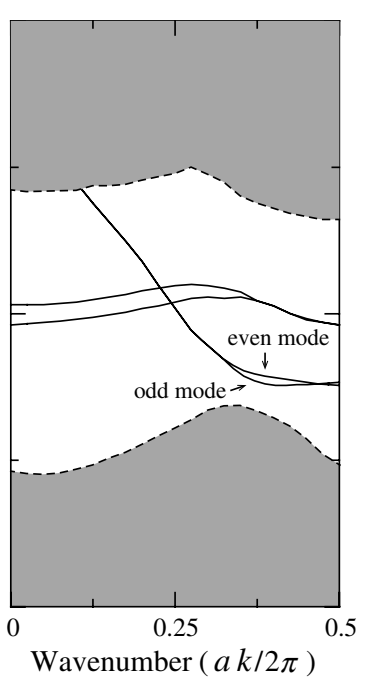

(b)
Fig. 6. Band diagrams of (a) a straight waveguide and (b) a directional coupler. The photonic crystal is a two-dimensional periodic structure having a lattice constant of $390 \mathrm{~nm}$ in a GaAs slab whose refractive index and thickness are 3.396 and $190 \mathrm{~nm}$, respectively. The slab is surrounded by air. The calculation was done using a three-dimensional finite-difference time-domain method. The experimentally estimated group velocity at $1337.66 \mathrm{~nm}$, which is obtained using the free spectral range (FSR) of the ring resonator, is indicated as the slope of the dotted line in (a). 


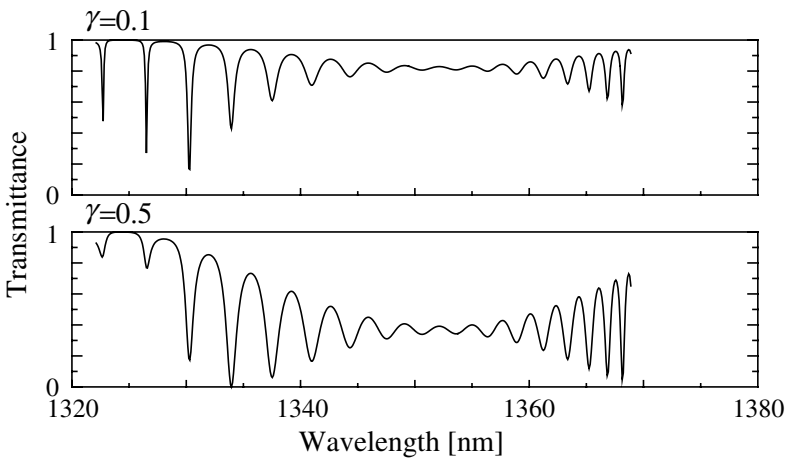

(a) $L_{\text {ring }}=280 a$

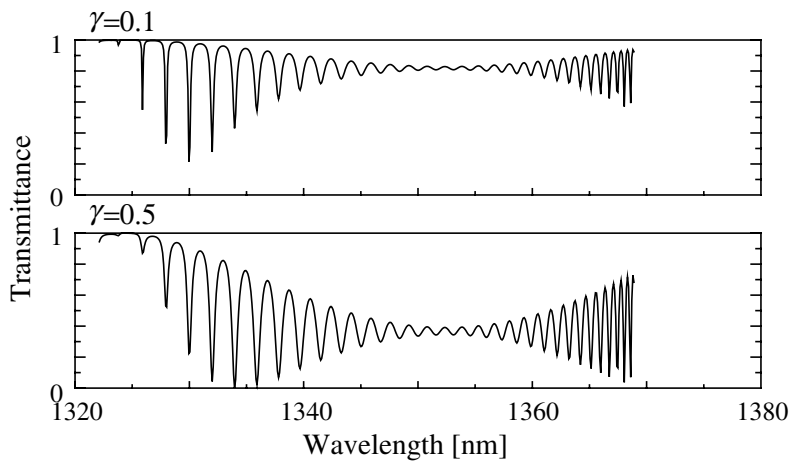

(b) $L_{\text {ring }}=520 a$

Fig. 7. Numerically calculated transmittance spectra for ring waveguides of length (a) $280 a$ and (b) $520 a$. The attenuation in the ring waveguide, $\gamma$ [see Eq. (1)], was set to 0.1 or 0.5 .

large loss, respectively, in the ring waveguide. We can see that the spectra have resonance peaks with a wavelength interval of $3.5 \mathrm{~nm}$ for $L_{\text {ring }}=280 a$ and $1.9 \mathrm{~nm}$ for $L_{\text {ring }}=520 a$, independent of $\gamma$. There is a possibility that there are resonance peaks that are not attributable to the ring resonance. One is a Fabry-Perot resonance between the input and the output ports of the device. Its distance is about $1.2 \mathrm{~mm}$. Since the resonant wavelength interval is approximately inversely proportional to the distance, the corresponding interval is $0.16 \mathrm{~nm}$. We can see these resonance peaks in Figs. $\underline{4}$ and $\underline{5}$ as small oscillations of the spectra. These resonance peaks are sufficiently small and can easily be identified. Another contribution is the Fabry-Perot resonance between two bending points in the ring waveguide. In the hexagonal ring resonator, the distances between two bending points are $80 a$ and $30 a$ (see Fig. 3), which correspond to resonant wavelength intervals of 6.1 and $16 \mathrm{~nm}$, respectively. The peaks indicated by arrows in Figs. 4 and 5 have intervals of 2.89 and $1.7 \mathrm{~nm}$, which agree with the wavelength interval of the simulated spectra, not with the Fabry-Perot resonance.

\section{4. $Q$ VALUE CHARACTERIZATION OF RING CAVITIES}

The $Q$ value, indicating the strength of confinement of a cavity, is determined from the transmission spectra as

$$
Q_{\mathrm{sys}}^{-1}=\frac{\text { FWHM of resonance peak }}{\text { peak wavelength }},
$$

where $Q_{\text {sys }}$ is the $Q$ value of the entire device, including the ring waveguide and the directional coupler, and is variable depending on the state of the directional coupler. The static $Q$ value exhibiting the strength of confinement of light in the ring waveguide is defined as $Q_{\text {ring }}$, and it will be discussed in the following section. We noticed two resonant peaks around (i) 1340 and (ii) $1351 \mathrm{~nm}$ (indicated by arrows in Figs. $\underline{4}$ and 5) that are clearly visible for both the hexagonal and the circular ring cavities. For the hexagonal ring resonator, the peak wavelengths were (i) 1340.15 and (ii) $1351.21 \mathrm{~nm}$, and their full-width at half-maximum (FWHM) values were (i) 0.67 and (ii) $0.86 \mathrm{~nm}$, respectively. From these values and Eq. (2), we found $Q_{\text {sys }}$ as (i) $2.0 \times 10^{3}$ and (ii) $1.6 \times 10^{3}$. In contrast, for the circular ring resonator, the peak wavelengths were (i) 1337.66 and (ii) $1351.14 \mathrm{~nm}$, and their FWHM were (i) 0.082 and (ii) $0.25 \mathrm{~nm}$. Then we found $Q_{\text {sys }}$ as (i) $1.6 \times 10^{4}$ and (ii) $5.3 \times 10^{3}$.

\section{DISCUSSION}

Compared with the $Q_{\text {sys }}$ values of the hexagonal ring resonator, those of the circular ring resonator exhibit high values. The difference in $Q$ values is due to the difference in radiation loss in the ring waveguides. The photographs in Figs. $\underline{4}$ and $\underline{5}$ are the top views of the ring cavities when light at a resonant wavelength (1337.59 $\mathrm{nm}$ for the hexagonal ring resonator and $1339.08 \mathrm{~nm}$ for the circular ring resonator) was input. Bright spots are observed at bending points of the hexagonal ring resonator (Fig. 4), demonstrating considerable loss of light. However, evaluation of the strength of confinement of light in the ring waveguide only from $Q_{\text {sys }}$ is not accurate. $Q_{\text {sys }}$ depends on light leakage from both the ring waveguide and the directional coupler. For example, even if the confinement of light in the ring waveguide is sufficiently strong, when the directional coupler is in the cross state, light in the ring waveguide immediately leaks out to the $\mathrm{I} / \mathrm{O}$ waveguide. In such a case, $Q_{\text {sys }}$ has a low value. In this work, we also estimated the $Q$ value of the ring waveguide excluding the optical coupler $\left(Q_{\text {ring }}\right)$ by the following method.

Optical energy $U$ in the ring waveguide decreases by a combination of coupling to the I/O waveguide and radiation losses with a time dependence of

$$
U(t)=U_{0} \exp \left(-\frac{\omega t}{Q_{\mathrm{sys}}}\right)
$$

where $U_{0}$ is the optical energy at $t=0$. If there is no optical coupling between the $\mathrm{I} / \mathrm{O}$ and the ring waveguide, the optical energy only decreases from radiation losses in the ring waveguide, and $U(t)$ is then given by

$$
U(t)=U_{0} \exp \left(-\frac{\omega t}{Q_{\text {ring }}}\right) .
$$

In contrast, if there is no radiation loss in the ring waveguide, the optical energy decreases because of the coupling with the $\mathrm{I} / \mathrm{O}$ waveguide, so that $U(t)$ is given by

$$
U(t)=U_{0} \exp \left(-\frac{\omega t}{Q_{\mathrm{DC}}}\right),
$$

where $Q_{\mathrm{DC}}$ is an imaginary $Q$ value of the entire device without radiation loss in the ring waveguide. In this case, the optical energy in the ring resonator decreases by the factor 


$$
1-\kappa=\exp \left(-\frac{2 \pi \omega_{n} T_{0}}{Q_{\mathrm{DC}}}\right)
$$

where $\omega_{n}$ is the normalized frequency, equal to $a \omega / 2 \pi c$, with $c$ the speed of light in vacuum, and $T_{0}$ is the period for light to travel around the ring waveguide once,

$$
T_{0}=\frac{L_{n}}{v_{g}}
$$

where $v_{g}$ is the group velocity normalized by $c$. The group velocity can be obtained from the free spectral range (FSR) of the resonant peak as

$$
v_{g}=L_{n} \times \mathrm{FSR}
$$

where $L_{n}$ is the length of the ring waveguide normalized by the lattice constant $a$. In Eq. (6), $\kappa$ is a coupling coefficient determined by the transmitted intensity in the bar direction $\left(I_{\mathrm{bar}}\right)$ and cross direction $\left(I_{\text {cross }}\right)$ as

$$
\kappa=\frac{I_{\text {cross }}}{I_{\text {bar }}+I_{\text {cross }}} .
$$

Actually, loss at the ring waveguide and the directional coupler exist simultaneously, and thus $U(t)$ must be

$$
U(t)=U_{0} \exp \left(-\frac{\omega t}{Q_{\mathrm{DC}}}\right) \exp \left(-\frac{\omega t}{Q_{\text {ring }}}\right),
$$

and it is equivalent to Eq. (3), yielding

$$
\frac{1}{Q_{\mathrm{sys}}}=\frac{1}{Q_{\text {ring }}}+\frac{1}{Q_{\mathrm{DC}}} .
$$

Therefore, $Q_{\text {ring }}$ can be obtained from measurements of $Q_{\text {sys }}$ and $Q_{\mathrm{DC}}$.

At first, we estimated $Q_{\text {ring }}$ of the hexagonal ring resonator. The resonant peaks in the hexagonal ring resonator are not clear, and therefore it is difficult to find the FSR. Here we assumed that the group velocities in the circularly bent waveguide and the straight waveguide at the same wavelength are equal, and we used the same group velocity as that for the circular ring waveguide. We have previously determined that the dispersion relations of waves propagating in an ordinary straight waveguide and a circularly curved waveguide coincide [20]. The FSR at peaks (i) and (ii) was calculated by the difference between adjacent peaks, and by using Eq. (8), we obtained group velocities of (i) 0.197494 and (ii) 0.176416 (normalized by the speed of light in vacuum). In general, the group velocity in a photonic crystal waveguide tends to be slow as the wavelength becomes longer, and this trend is consistent with the measured result. The group velocity is equivalent to the slope of the dispersion curves of the propagation mode. The dotted line in Fig. 6(a) is a dispersion relation that has the experimentally estimated group velocity at (i) $1337.66 \mathrm{~nm}$. The group velocity obtained by the band diagram is 0.217143 . The error of about $9.0 \%$ may be due to the error in the FSR that results from dispersion properties in branch waveguides or Fabry-Perot resonance between the bent or the branch waveguides. The coupling efficiency $\kappa$ was calculated from the spectra of a single directional coupler. At the wavelengths of (i) and (ii), $\kappa$ was 0.396197 and 0.737357 , respectively. From the values obtained above, we can find $Q_{\mathrm{DC}}$ as (i) 5148.00 and (ii) 2153.01. These values show that if optical coupling is larger [(i) $0.39617<$ (ii) 0.737357], $Q_{\text {DC }}$ becomes smaller [(i) $5148.00>$ (ii) 2153.01]. This trend is consistent with the definition of $Q_{\mathrm{DC}}$. Finally, from Eq. (11), we found $Q_{\text {ring }}$ as (i) $3.2 \times 10^{3}$ and (ii) $5.9 \times 10^{3}$.

Next, we also estimated the $Q_{\text {ring }}$ of the circular ring resonator. The coupling efficiency $\kappa$ was calculated from the spectra of a single directional coupler. At the peak wavelengths of (i) and (ii), $\kappa$ was 0.131992 and 0.386379 , respectively. From the values obtained above, we can find $Q_{\mathrm{DC}}$ as (i) 34074.5 and (ii) 10946.0. Finally, from Eq. (11), we found $Q_{\text {ring }}$ as (i) $3.1 \times 10^{4}$ and (ii) $1.0 \times 10^{4}$.

Finally, we modify $Q_{\text {ring }}$ by considering the effect of the difference in length of the ring waveguide. Here we assume the loss in the straight waveguides is sufficiently less than that in the bent waveguides. A photonic crystal slab waveguide confines propagating light by total reflection and its photonic bandgap, and therefore the loss is nearly zero unless there are structural defects. However, both sharply and circularly bent waveguides have no crystalline structure, due to their asymmetric bent line defects or bent air hole arrays. This means that the photonic bandgap might disappear, and even though there are no structural defects, propagation loss necessarily occurs. Under this assumption, $Q_{\text {ring }}$ of a hexagonal ring waveguide is determined by the number of times that the propagating light passes through the apexes of the hexagonal ring waveguide (leaking spots) per unit time. Therefore, $Q_{\text {ring }}$ is proportional to the length of the ring waveguide. Since the lengths of the waveguide of the measured hexagonal and circular ring waveguides are $280 a$ and $520 a$, respectively, we can estimate $Q_{\text {ring }}$ of the $520 a$-long hexagonal ring waveguide by multiplying by $520 / 280$. As a result, we found the modified $Q_{\text {ring }}$ as (i) $6.0 \times 10^{3}$ and (ii) $1.1 \times 10^{4}$. From the result, the estimated $Q_{\text {ring }}$ of the circular ring waveguide at wavelength (i) is 5.2 times as high as that of the hexagonal ring waveguide. However, $Q_{\text {ring }}$ at wavelength (ii) is comparable. We discuss the difference in terms of the loss in the bent waveguide. The optical intensity after circulating the ring waveguide can be expressed by

$$
U_{6}=U_{0} \exp \left(-\frac{\omega T_{1}}{Q_{\text {ring }}}\right)
$$

where $T_{1}=L / v_{g}$ and $U_{0}$ are the time to circulate the ring waveguide and the incident energy, respectively. $U_{6} / U_{0}$ corresponds to the transmittance of a waveguide having $6 \times 60^{\circ}$ bent points. From the transmittance, we can estimate the transmittance of $2 \times 60^{\circ}$ bent waveguides as shown Fig. 2 . By assuming that each bent point has equal transmittance, the transmittance $U_{2}$ of the $2 \times 60^{\circ}$ bent waveguides can be expressed as $U_{2}=U_{6}^{1 / 3}$. The transmittance was obtained from the transmitted intensity of a bent waveguide normalized by that of a straight waveguide. $U_{2}$ values at wavelength (i) are 0.76531 for the hexagonal ring waveguide and 0.94791 for the circular ring waveguide, whereas $U_{2}$ values at wavelength (ii) are 0.8504 for the hexagonal ring waveguide and 0.8367 for the circular ring waveguide. Evaluating the quantitative agreement of $U_{2}$ is difficult because of the variation of 
transmittance between fabricated devices. The transmitted intensity of the sharply bent waveguide for Fig. 2 may be lower than that for the bent waveguide in the hexagonal ring waveguide. However, the experimentally obtained $U_{2}$ at (i) is increased by replacing the sharply bent waveguide by a circularly bent waveguide, whereas it is comparable at (ii), and this trend is consistent with $U_{2}$ estimated from $Q_{\text {ring. }}$. As we can see from the spectrum of the circular ring resonator (Fig. 5), the transmittance rapidly decreases as wavelength becomes longer. This property comes from circularly bent waveguides, which can also be numerically simulated as the radiation toward the inner side of the curvature. To inhibit such radiation, we are redesigning the circularly bent waveguide by using its photonic band diagrams.

Not only does the circular ring resonator improve the strength of the confinement of light, but it also improves the conservation of optical pulse shape. Resonance peaks in the spectrum of a hexagonal ring resonator in a range of more than $1355 \mathrm{~nm}$ are randomly oscillating, and it is difficult to identify a resonant peak, whereas the numerically simulated spectrum (Fig. 7) has nearly periodic resonance peaks. This is because the Fabry-Perot resonance in the ring waveguide is increased at the longer wavelength side. As we can see from the transmitted intensity of the waveguide having sharp bends, as shown in Fig. 2, the fluctuation in the spectrum also becomes larger as the wavelength becomes longer. The hexagonal ring waveguide, which consists of the same bent waveguide, also exhibits such a Fabry-Perot resonance in the same way. Furthermore, the six sharply bent points provide many Fabry-Perot resonances with different FSR values, resulting in irregular fluctuation in the spectrum. These resonances, which are caused by the increased backreflection at the bent point of the bent waveguide, cause an unexpected frequency property in the transmittance, resulting in the transformation of a propagating pulse shape.

However, compared with the hexagonal ring resonator, the spectrum of the circular ring resonator in Fig. $\underline{5}$ has nearly periodic resonance peaks, as shown by the simulated spectra in Fig. 7, and, in addition, each resonance peak is clearly identifiable. This improvement shows that the undesired Fabry-Perot resonances in the ring waveguide are removed. Consequently, the replacement of the waveguide is also effective for conserving the pulse shape stored in the ring waveguide.

\section{CONCLUSION}

For the purpose of developing an integrated optical buffer memory based on a photonic crystal ring resonator, a ring resonator with an improved $Q$ value was designed and fabricated. Previously observed low $Q$ values stem from radiation losses at bending points in the waveguide. To improve the optical confinement, we replaced the $60^{\circ}$ bent waveguide with a circularly curved waveguide. The resulting $Q$ values for the conventional ring resonator and for the circular ring resonator are $2.0 \times 10^{3}$ and $1.6 \times 10^{4}$, respectively. We found an eightfold increase in the $Q$ value. Since these $Q$ values contain the loss by coupling to the I/O waveguide, we estimated $Q$ values that eliminated the coupling. The estimated values are $6.0 \times 10^{3}$ for the hexagonal ring resonator and $3.1 \times 10^{4}$ for the circular one, demonstrating a 5.2-fold improvement. This result shows that a significantly high confinement of light can be achieved.
However, at the longer wavelength side (1351 mm), there was only slight improvement. This is because the transmittance of the circularly bent waveguide is lower. We need to improve this transmittance and redesign the waveguide in future work. Around the cutoff frequency at the longer wavelength side, the resonance peaks became clear and identifiable by the replacement of the bent waveguide, due to the reduction of unexpected Fabry-Perot resonances in the ring waveguide. Replacing the bent waveguide is effective for the conservation of the pulse shape stored in the ring waveguide. Furthermore, since the wavelength region that provides slow light is important for device miniaturization, improvement of the resonance property in that region is also a significant achievement.

\section{ACKNOWLEDGMENTS}

This work was supported by the New Energy and Industrial Technology Development Organization (NEDO; no. 08C46215a) and the Japan Society for the Promotion of Science (JSPS) KAKENHI (Grant-in-Aid for JSPS Fellows, no. 2242).

\section{REFERENCES}

1. R. Langenhorst, M. Eiselt, W. Pieper, G. Grosskopf, R. Ludwig, L. Kuller, E. Dietrich, and H. G. Weber, "Fiber loop optical buffer,” J. Lightwave Technol. 14, 324-335 (1996).

2. E. Yablonovitch, "Inhibited spontaneous emission in solid-state physics and electronics," Phys. Rev. Lett. 58, 2059-2062 (1987).

3. S. John, "Strong localization of photons in certain disordered dielectric superlattices,” Phys. Rev. Lett. 58, 2486-2489 (1987).

4. D. Rafizadeh, J. P. Zhang, S. C. Hagness, A. Taflove, K. A. Stair, S. T. Ho, and R. C. Tiberio, "Waveguide-coupled AlGaAs/GaAs microcavity ring and disk resonators with high finesse and $21.6 \mathrm{~nm}$ free spectral range," Opt. Lett. 22, 1244-1246 (1997).

5. B. E. Little, J. S. Foresi, G. Steinmeyer, E. R. Thoen, S. T. Chu, H. A. Haus, E. P. Ippen, L. C. Kimerling, and W. Greene, "Ultracompact $\mathrm{Si}-\mathrm{SiO} 2$ microring resonator optical channel dropping filters," IEEE Photon. Technol. Lett. 10, 549-551 (1998).

6. A. Yariv, "Universal relations for coupling of optical power between microresonators and dielectric waveguides," Electron. Lett. 36, 321-322 (2000).

7. S. T. Chu, B. E. Little, W. Pan, T. Kaneko, S. Sato, and Y. Kokubun, "An eight-channel add-drop filter using vertically coupled microring resonators over a cross grid," IEEE Photon. Technol. Lett. 11, 691-693 (1999).

8. S.-H. Kim, H.-Y. Ryu, H.-G. Park, G.-H. Kim, Y.-S. Choi, and Y.-H. Lee, "Two-dimensional photonic crystal hexagonal waveguide ring laser," Appl. Phys. Lett. 81, 2499-2501 (2002).

9. K. Furuya, N. Yamamoto, Y. Watanabe, and K. Komori, "Novel ring waveguide device in a $2 \mathrm{D}$ photonic crystal slab: transmittance simulated by finite-difference time-domain analysis," Jpn. J. Appl. Phys. 43, 1995-2001 (2004).

10. M. David, F. Monifi, A. Ghaffari, and M. S. Abrishamian, "Heterostructure wavelength division demultiplexers using photonic crystal ring resonators," Opt. Commun. 281, 4028-4032 (2008).

11. S. Kim, J. Cai, J. Jiang, and G. P. Nordin, "New ring resonator configuration using hybrid photonic crystal and conventional waveguide structures," Opt. Express 12, 2356-2364 (2004).

12. D. Goldring, U. Levy, and D. Mendlovic, "Highly dispersive micro-ring resonator based on one dimensional photonic crystal waveguide design and analysis," Opt. Express 15, 3156-3168 (2007).

13. M. Tokushima and H. Yamada, "Photonic crystal line defect waveguide directional coupler," Electron. Lett. 37, 1454-1455 (2001).

14. S. Boscolo, M. Midrio, and C. Someda, "Coupling and decoupling of electromagnetic waves in parallel $2 \mathrm{D}$ photonic crystal waveguides," IEEE J. Quantum Electron. 38, 47-53 (2002).

15. N. Yamamoto, T. Ogawa, and K. Komori, "Photonic crystal directional coupler switch with small switching length and wide bandwidth," Opt. Express 14, 1223-1229 (2006). 
16. S.-H. Jeong, N. Yamamoto, J. Sugisaka, M. Okano, and K. Komori, "GaAs-based two-dimensional photonic crystal slab ring resonator consisting of a directional coupler and bent waveguides," J. Opt. Soc. Am. B 24, 1951-1959 (2007).

17. S.-H. Jeong, J. Sugisaka, N. Yamamoto, M. Okano, and K. Komori, "Resonant characteristics in a two-dimensional photonic crystal ring resonator with a triangular lattice of air holes," Jpn. J. Appl. Phys. 46, L534-L536 (2007).

18. A. Mekis, J. C. Chen, I. Kurland, S. Fan, P. R. Villeneuve, and J. D. Joannopoulos, "High transmission through sharp bends in photonic crystal waveguides," Phys. Rev. Lett. 77, 3787-3790 (1996).

19. M. H. Shih, W. J. Kim, W. Kuang, J. R. Cao, S.-J. Choi, J. D. O'Brien, and P. D. Dapkus, "Experimental characterization of the reflectance of $60^{\circ}$ waveguide bends in photonic crystal waveguides," Appl. Phys. Lett. 86, 191104 (2005).

20. J. Sugisaka, N. Yamamoto, M. Okano, K. Komori, T. Yatagai, and M. Itoh, "Development of curved two-dimensional photonic crystal waveguides," Opt. Commun. 281, 5788-5792 (2008).

21. N. Ikeda, H. Kawashima, Y. Sugimoto, T. Hasama, K. Asakawa, and H. Ishikawa, "Coupling characteristic of micro planar lens for 2D photonic crystal waveguides," in Proceedings of the IEEE 19th International Conference on Indium Phosphide and Related Materials (IEEE, 2007), pp. 484-486.

22. L. F. Stokes, M. Chodorow, and H. J. Shaw, "All-single-mode fiber resonator," Opt. Lett. 7, 288-290 (1982). 